\title{
Les défis du système éducatif algérien dans le droit à l'éducation et la concrétisation de l'égalité : Etude descriptive et analytique des indicateurs de l'enseignement obligatoire
}

Dr.Zoulikha Toutaoui,Université de Tizi Ouzou.

\section{Résumé:}

L'Algérie a hérité après l'indépendance une situation grave dans le domaine de l'éducation représentée dans le taux d'analphabétisme de plus de $85 \%$, de la faible proportion des inscrits par rapport aux besoins et aspirations de la communauté. Puis vint la phase du projet de réforme globale du système éducatif en Avril 1976, et a procédé à l'application de l'école fondamentale à partir de 1980. La deuxième nouvelle reforme associé à des changements que connaît le pays, Parmi les piliers de la réforme, la dimension démocratique à l'éducation, l'éducation gratuite, l'égalité des chances pour toutes les catégories, adapter l'éducation à la majorité des apprenants ,assurer les différences et les diversités, assurer l'enseignement spécialisé pour les enfants aux besoins particuliers. De ces efforts déployés dans le secteur de l'éducation par les différentes réformes, on s'interroge comment ils se reflètent dans la réalité sur terrain? Pour répondre, nous avons procédé à une étude analytique et descriptive sur le système d'enseignement obligatoire en Algérie, en se concentrant sur le droit à l'enseignement et l'égalité des chances entre les sexes et entre les régions.

الملذهص

ورثت الجزائر بعد الاستقلال وضعا خطيرا في مجال التعليم مثلة في نسبة الأمية أكثر من 85

\%، و انخفاض نسبة التسجيلات مقارنة باحتياجات و تطلعات المجتمع . ثم جاءت مرحلة الإصلاح الشامل لنظام التعليم في افريل 1976، وشرع في تطبيق المرحلة الأساسية ابتداء من 1980 ـ الاصلاح

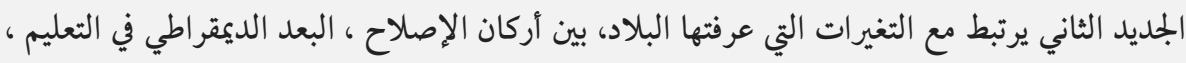

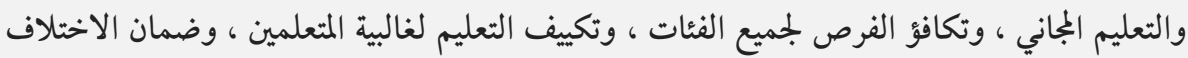
و التنوع ، وضمان التربية الخاصة للأطفال ذوي الاحتياجات الخاصة ـ من مذ هذه الجهود الكبع الكبيرة في قطاع

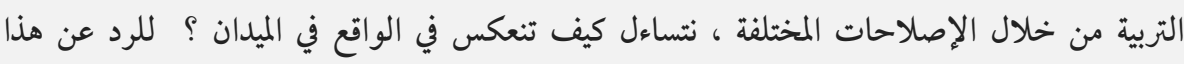
السؤال أجرينا دراسة وصفية وتحليلية لنظام التعليم الإلزامي في الجزائر ، مع التركيز على الحق في التعليم وتكافؤ الفرص بين الجنسين و بين المناطق. 


\section{Introduction :}

Au lendemain de son indépendance, l'Algérie a hérité d'une situation catastrophique en matière d'éducation, le taux d'analphabétisme dépassait $85 \%$ et le taux de scolarisation était très faible par rapport aux besoins et aux aspirations de la société; un système éducatif étranger, contenu étranger, organisation et objectifs étrangers; des capacités très limitées et un manque d'encadrement technique et administratif au niveau national.

La première période postindépendance a été caractérisée par une forte demande d'éducation, de formation et de promotion sociale et culturelle. Le droit à l'éducation et à la formation était la première priorité ; la scolarisation a connu une évolution impressionnante, que ce soit en nombre ou en capacités d'accueil ou encore en ce qui concerne l'encadrement. L'école algérienne a vécu une constante évolution, imposée par la nécessité d'accueillir les nombres sans cesse grandissants d'élèves, et garantir une place pour chaque membre et ce par le recrutement, la formation et la construction continue.

Les premières réformes, caractérisées par la fixation des priorités et la définition des objectifs de l'école fondamentale, ont été une mise en œuvre des choix nationaux, tel que la démocratisation de l'enseignement, et ont mené à une augmentation du taux de scolarisation de $20 \%$ en 1962 jusqu'à $69 \%$ en 1972, avec un relatif 
équilibre concernant la répartition géographique des établissements d'éducation dont le nombre a dépassé 7000 établissements en 1972 contre 3360 en 1962. (1)

Suit la période des réformes globales du système éducatif au mois d'avril 1976 :

- Enseignement préparatoire non obligatoire

- Enseignement fondamental polyvalent obligatoire pour 9 ans.

- Enseignement secondaire, comprenant un enseignement secondaire général débouchant à l'enseignement supérieur, un enseignement secondaire spécialisé, un enseignement secondaire technique et professionnel préparant à la vie professionnelle et qui permet aussi de passer à la formation supérieure (2)

Leur application a commencé à partir de 1980 et se sont distinguées par :

- La généralisation de l'enseignement fondamental pour 9 années.

- La démocratisation de l'admission à l'enseignement secondaire et à l'enseignement supérieur

- Mise en place d'un dispositif de formation professionnelle et d'apprentissage indépendant.

- Augmentation du nombre d'élèves dans le système éducatif suite à la croissance démographique. (3)

La période allant de 1992 jusqu'à 2000 a été caractérisée par la phase de complémentarité entre les différents systèmes éducatifs de base , 
avec leurs défaillances et carences, entre autres, le manque d'écoles maternelles destinées aux enfants de 4 à 6 ans, non obligatoires mais prévues par l'ordonnance $\mathrm{n}^{\circ} 76-35$ (décision d'application $n^{\circ} 70-76$ ), pour les écoles maternelles et jardins d'enfants, absence d'enseignement spécialisé destiné aux enfants souffrant de difficultés scolaires, pourtant prévu par l'ordonnance 35 - 19 avril 1976.(4)

L'absence de ces deux types enseignements a eu des conséquences sur les résultats pédagogiques, suite aux autres réformes dans l'enseignement secondaire et la formation professionnelle.

L'intérêt a été porté sur la formation continue. En dépit de l'évolution peu importante qu'a connue cette formation dans chaque sous-secteur, elle a fourni des capacités importantes en matière de formation. Elle ne représente pas un secteur ou un système homogène; c'est un ensemble d'établissements chargés de fournir des services.

Les statistiques de 1992 ont fait état de ce qui suit :

- Centre nationale d'enseignement généralisé : Dispense une formation par correspondance pour environ 65000 élèves, dont $27 \%$ en enseignement fondamental et $68 \%$ en enseignement secondaire et $5 \%$ assistent aux examens professionnels pédagogiques et de gestion. 
- Centre nationale de formation professionnelle par correspondance : prend en charge 41500 élèves, plus 4300 élèves suivent les cours de soir de formation professionnelle.

- Université de formation continue : Dispense une formation pour 13000 étudiants pour la phase pré-universitaire, et 14000 étudiants pour la phase graduation; elle vise les travailleurs et les adultes et procure également des formations spéciales selon les demandes des différents secteurs et entreprises industrielles.

- Lutte contre l'analphabétisme : Le centre national de lutte antianalphabétisme a été créé en 1964 pour encourager la lutte contre l'analphabétisme, en fournissant un soutien technique et pédagogique et en formant les illettrés. Le centre a été placé sous tutelle du ministère de l'éducation nationale. Le programme a commencé vers la fin des années soixante en collaboration avec l'organisation de l'Unesco et le programme de développement des Nations Unis. Ce programme a touché au départ 100000 travailleurs comme première expérience, et après 8 ans le programme a touché $50 \%$ de cette catégorie. Durant les deux premiers plans quadriennaux, le programme de lutte contre analphabétisme a pris en charge 550000 personnes, et après l'application de l'école fondamentale en 1980 le ministère de l'éducation a légèrement abandonné cette activité. Mais pendant ces dernières années l'activité a ravivé grâce aux tentatives des associations et à l'intérêt 
porté par les organisations internationales au problème de l'analphabétisme et au droit à l'éducation pour tous. (5)

De nouvelles réformes ont été engagées ; elles étaient les deuxièmes réformes du genre après les réformes profondes des années 70. Les réformes des années 70 ont été imposées par la période postindépendance, où la priorité a été donnée à l'authentification de l'école dans ses contenus, cadres et programmes, et à sa démocratisation et son ouverture sur les sciences et les technologies. Quant aux nouvelles réformes, elles étaient imposées par d'autres circonstances liées essentiellement aux changements qu'a vécus le pays dans les domaines économique, politique et social et aussi par les défis de la mondialisation qui touche différents domaines. (6)

Les nouvelles réformes ont été marquées par la technologie de l'information et de la communication et ont commencé à opérer des changements dans les moyens et méthodes d'enseignement. Elles ont été précédées par des préparations intensives avant de les mettre en œuvre pendant l'année scolaire 2003/2004. Des modifications ont été introduites dans les textes de l'ordonnance de 1976, telle que « L'enseignement est gratuit à tous les niveaux, dans les établissements du secteur public» «introduction de l'enseignement de la langue amazigh dans le système éducatif » «Le système éducatif est du ressort de l'Etat», toutefois, tout personne qualifié, morale ou physique, soumise au droit privé, peut 
créer un établissement d'enseignement privé. L'établissement d'enseignement privé doit appliquer les programmes officiels enseignés dans les établissements publics relavant du ministère de l'éducation nationale.(7)

A travers cette présentation des étapes essentielles qu'a vécues le système éducatif en Algérie, on constate que les efforts consentis par l'Etat dans les différents secteurs éducatifs relevant de son ressort, étaient énormes :

Mais quels sont les résultats réalisés sur le terrain ? Et à partir de là nous avons posé les questions suivantes :

- Tout enfant algérien à l'âge de scolarisation a-t-il une place à l'école ou est-il hors de l'établissement scolaire?

- Quel est le taux d'admission des enfants au primaire?

- Y a-t 'il une parité des chances au droit à l'enseignement entre garçons et filles?

- Quel est le taux de scolarisation dans les établissements d'enseignement obligatoire? Comment ce taux a évolué à travers le temps?

- Y a-t 'il une parité du droit à l'enseignement entre les wilayas (régions)?

Pour répondre à toutes ces questions nous avons mené une étude descriptive et analytique au sujet du système éducatif obligatoire en Algérie. 


\section{Plan de l'étude :}

L'étude s'articule sur le droit à l'enseignement et la concrétisation de l'égalité entre les deux sexes et entre les régions dans l'enseignement obligatoire (primaire et moyen). Cette étude se veut descriptive et analytique en s'appuyant sur les indicateurs de l'enseignement appliqués dans plusieurs pays, qui nous révéleront à quel point l'égalité entre les deux sexes est respectée et nous révélerons également le droit des enfants algériens à l'enseignement et à la scolarisation dans les établissements d'enseignement primaire et dans les établissements d'enseignement moyen.

Les indicateurs sont :

\section{1- Indicateur du taux d'admission à l'enseignement primaire : c'est} le nombre de nouveaux élèves admis à l'école pour la première fois par rapport à l'ensemble de la population scolarisable (6 ans), c'està-dire ceux ayant droit à la scolarisation. Cet indicateur est subdivisé en :

- Indicateur du taux brut d'admission: c'est le nombre de nouveaux élèves admis à l'école pour la première fois (abstraction faite de leur âge) par rapport à l'ensemble de la population scolarisable (6 ans).

- Indicateur du taux net d'admission : c'est le nombre de nouveaux élèves admis à l'école pour la première fois ayant atteint l'âge de 6 ans par rapport à l'ensemble de la population scolarisable (6 ans). 
2- Indicateur du taux de scolarisation: c'est le nombre de la population scolarisée dans l'enseignement obligatoire par rapport au nombre de la population scolarisable (la catégorie des enfants doit être incluse dans ce système éducatif 6-15 ans). Cet indicateur est subdivisé en :

- Indicateur du taux brut de scolarisation : c'est le nombre de la population scolarisée à l'enseignement obligatoire (abstraction faite de leur âge) par rapport à la population scolarisable de la catégorie d'âge (6-15 ans).

- Indicateur du taux net de scolarisation : c'est le nombre de la population scolarisée de la catégorie d'âge (6-15 ans) par rapport par rapport à la population scolarisable de la catégorie d'âge (6-15 ans).

3- Indice de parité entre sexes : un indicateur consacré au taux d'admission des filles au primaire par rapport au taux d'admission des garçons (taux des filles/ taux des garçons). L'indice de parité est consacré au taux de scolarisation des filles par rapport au taux de scolarisation des garçons (taux des filles/ taux des garçons).(8)

4- Indicateur du coefficient de scolarisation : c'est le nombre de la population scolarisée par wilaya (dans l'enseignement primaire et dans l'enseignement moyen) par rapport à l'ensemble de la population de chaque wilaya.(9) (10) 


\section{Résultat de l'étude :}

A travers l'analyse des résultats des indicateurs d'éducation choisis, qui montrent le taux d'admission des enfants à l'enseignement, l'évolution du nombre de la population scolarisée dans l'enseignement obligatoire selon les sexes, le coefficient de scolarisation dans l'enseignement obligatoire, la disparité entre les wilayas (régions), nous avons procédé à une comparaison à travers le temps et avec certains pays, et nous avons obtenus les résultats suivants :

\section{1- Taus d'admission au primaire :}

\subsection{Taux brut d'admission au primaire :}

Le taux brut d'admission représente l'ensemble des nouveaux admis à l'école pour la première fois (abstraction faite de leur âge à leur admission, à 05 ans, avant l'âge légal d'admission et à 06 ans et plus) par rapport à l'ensemble de la population scolarisable (06 ans).

Graph (1) : Evolution du taux brut d'admission au primaire et indice de parité entre sexes 


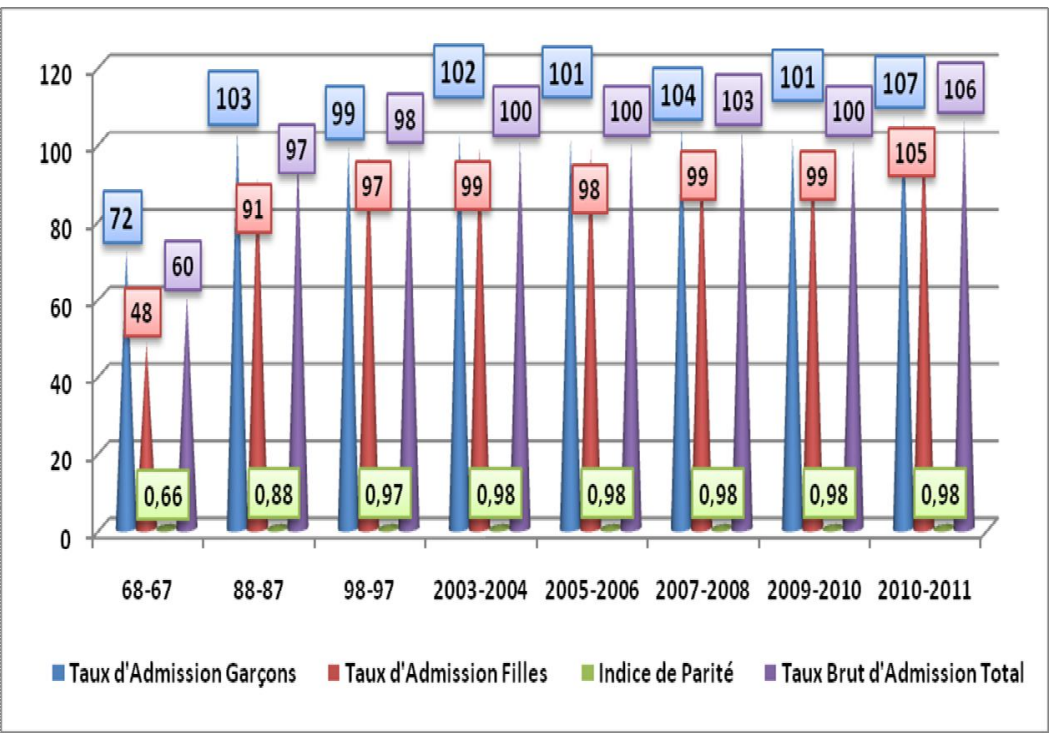

Source : Ministère de l'éducation nationale, (11) (12) (13) (14)

Comparaison à travers le temps : le nombre des nouveaux élèves a connu une importante et permanente évolution suite à la généralisation de l'enseignement de base pour tous les enfants durant les premières années postindépendance. Le taux brut d'admission au primaire a augmenté de $60 \%$ à $106 \%$, le taux d'admission des filles a également augmenté de $48 \%$ à $105 \%$ ainsi le taux d'admission des garçons qui a augmenté de $72 \%$ à $107 \%$. L'indice de parité indique qu'au début des années soixante la scolarisation des filles était faible par rapport à la scolarisation des garçons ; 50 \% des filles scolarisable ne rejoignaient pas les écoles. 
Comparaison entre Etats : nous avons effectué une comparaison entre les taux bruts d'admission enregistrés en Algérie et ceux enregistrés dans certains pays, pour montrer les disparités qui existent entre eux, et ce en optant pour l'année scolaire 2009-2010, et nous avons fait ressortir ce qui suit :

Graph (2) : Comparaison des taux d'admissions au primaire et indice de parité en Algérie et certains pays.

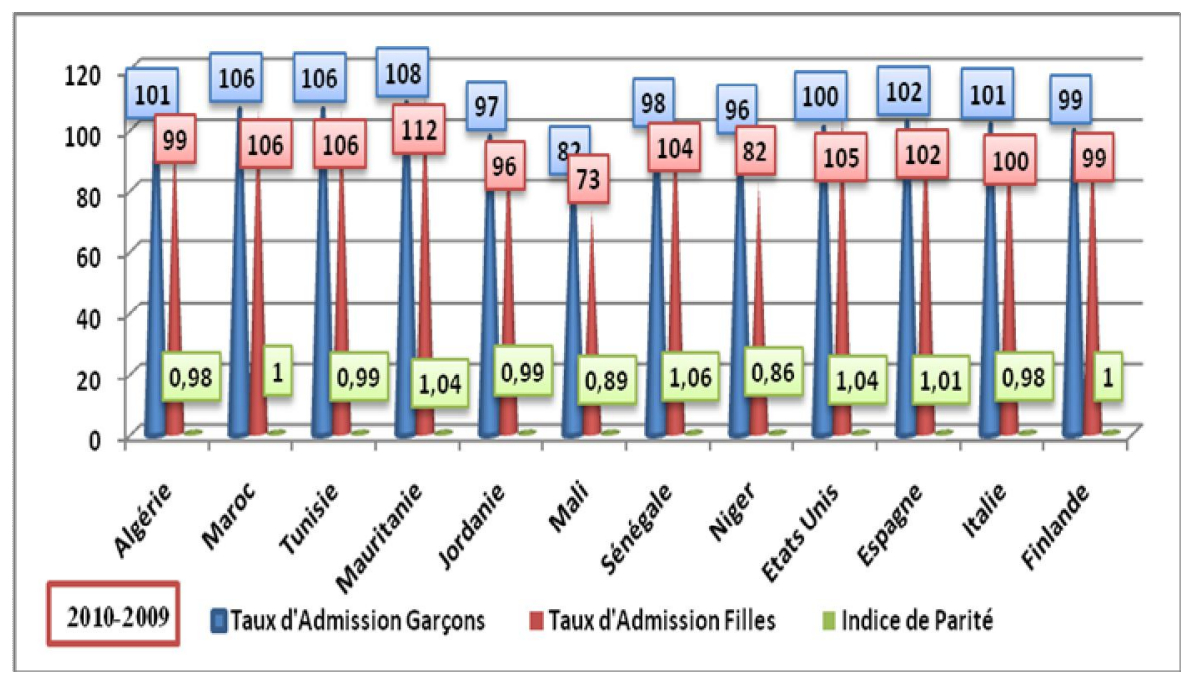

Source : Unesco (site UNESCO.org) (15)

A travers cette comparaison nous constatons qu'en Algérie le taux brut d'admission des filles au primaire est moindre par rapport aux taux enregistrés dans certains pays arabes et européens mais mieux que ceux enregistrés au Niger, Mali et Jordanie. Il en va de même pour l'indice de parité entre sexes. 
2.1 Taux net d'admission au primaire : Cet indicateur représente l'ensemble des nouveaux admis à l'école pour la première fois (à l'âge légal «06 ans ») par rapport au nombre d'enfants algériens à l'âge de 06 ans.

Graph (3): Evolution du taux net d'admission au primaire et indice de parité entre sexes.

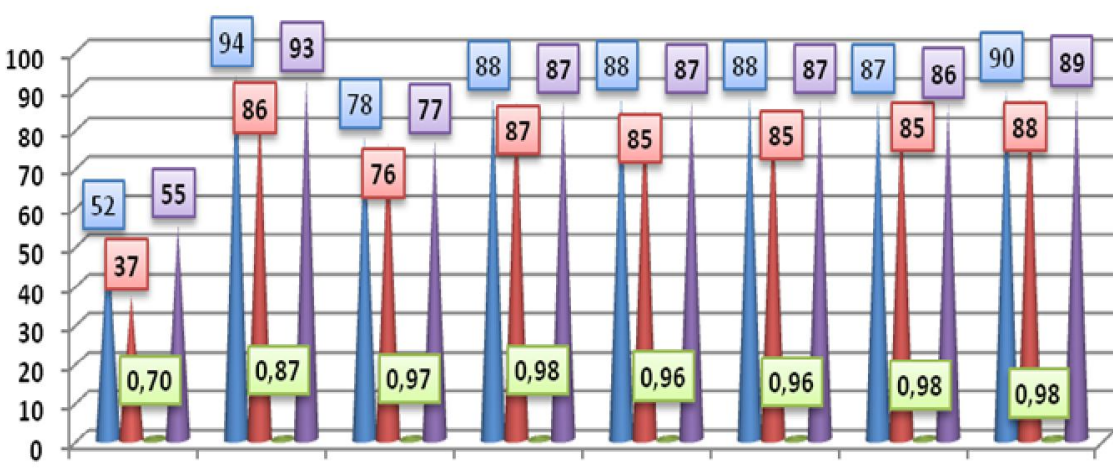

$\begin{array}{llllllll}68-67 & 88-87 & 98-97 & 2003-2004 & 2005-2006 & 2007-2008 & 2009-2010 & 2010-2011\end{array}$

- Taux d'Admission Garçons - Taux d'Admission Filles Indice de Parité — Taux Net d'Admission Total

Source : Ministère de l'éducation nationale (16) (17)(18) (19)

Comparaison à travers le temps : Comme nous l'avons signalé auparavant, le nombre des nouveaux élèves était en constante évolution après l'indépendance ; le taux brut d'admission de nouveaux élèves aux différentes âges était énorme; tandis que le taux net d'admission, c'est-à-dire le taux des enfants ayant atteint l'âge de scolarisation seulement, a augmenté de $55 \%$ à $89 \%$, le taux 
d'admission des filles est passé de $37 \%$ à $88 \%$, le taux d'admission des garçons est passé de $52 \%$ à $90 \%$. Il ressort du taux net d'admission durant les années 80 que $14 \%$ des filles à l'âge de 06 ans n'étaient pas admises aux écoles, pourtant l'indice de parité entre sexes a augmenté à 0,87 .

Comparaison entre Etat: Nous avons effectué une comparaison entre les taux nets d'admissions enregistrés en Algérie et ceux enregistrés dans certains pays, pour montrer les disparités qui existent entre eux, et ce en optant pour l'année scolaire 2009-2010, et nous avons fait ressortir ce qui suit :

Graph (4) : Comparaison des taux nets d'admission au primaire et indice de parité en Algérie et certains pays

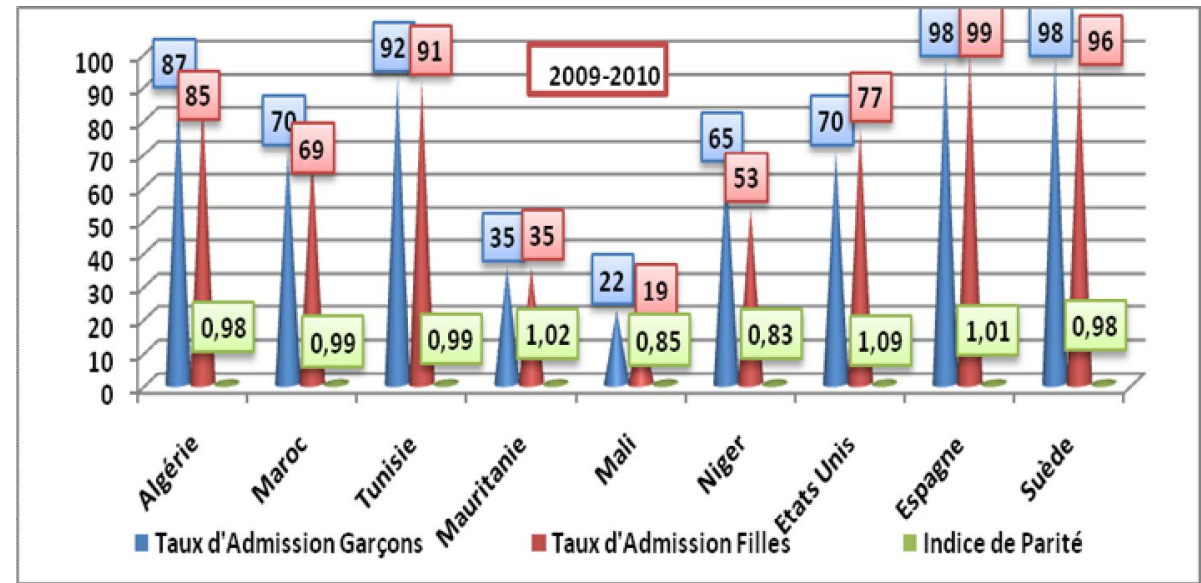

Source : Unesco (site UNESCO.org) (20)

A travers cette comparaison nous constatons qu'en Algérie le taux net d'admission des filles au primaire est mieux que celui du Maroc, des 
Etats unis, du Niger, du Mali, et de Mauritanie et moindre par rapport aux taux enregistrés en Tunisie, Espagne et Suède.

\section{2- Taux brut de scolarisation :}

\subsection{Taux brut de scolarisation au primaire :}

Cet indicateur représente le nombre de la population scolarisée au primaire quel que soit leur âge (suivant les données fournies par les statistiques du ministère de l'éducation nationale, leur âge varient entre 5 et 15 ans) par rapport à la population algérienne de la catégorie d'âge de 06 à 11 ans représentant le nombre d'années passées par un élève au primaire. Les résultats de cet indicateur sont les suivants :

Graph (5): Evolution du taux brut de scolarisation au primaire et indice de parité entre sexes

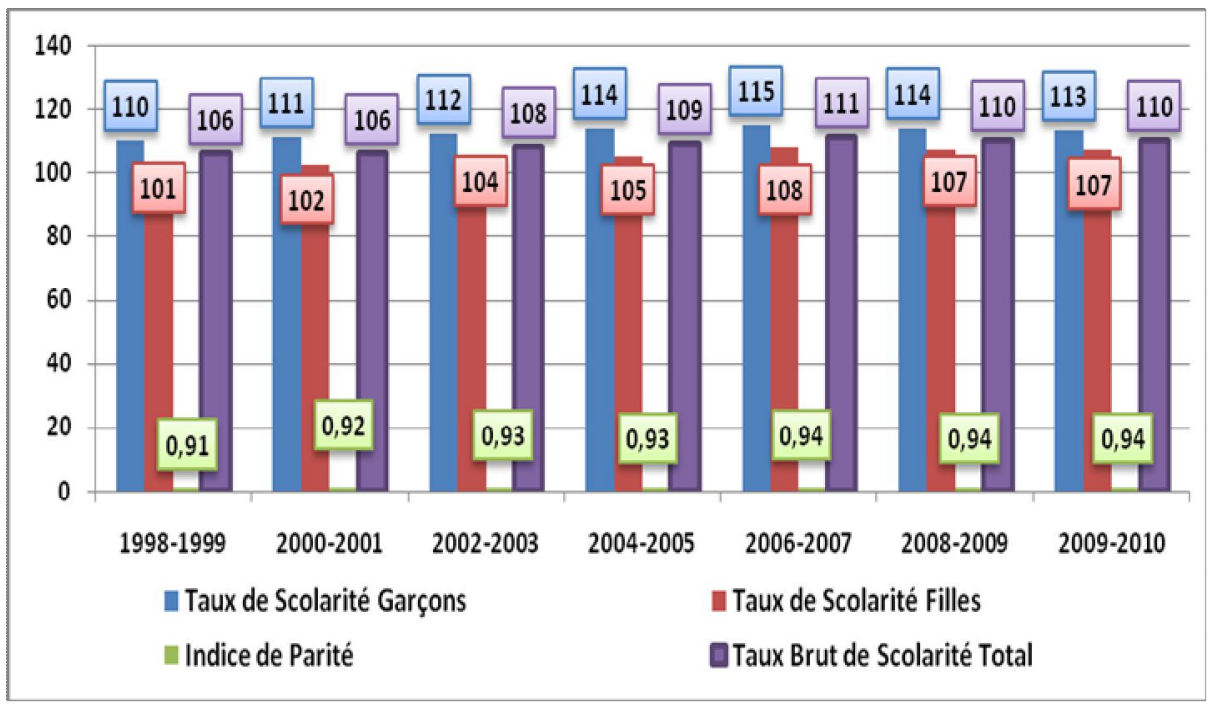

Source : Ministère de l'éducation nationale (21) (22) (23) (24) 
Comparaison à travers le temps : La scolarisation des enfants au primaire est en constante augmentation ces dernières années. Le taux brut de scolarisation oscille entre $106 \%$ et $\quad 110 \%$ durant la période allant de 1998 à 2010 ; le taux de scolarisation des filles a augmenté de $101 \%$ à $107 \%$ et le taux de scolarisation des garçons de $110 \%$ à $113 \%$. L'indice de parité entre sexes a connu une augmentation de 0.91 à 0.94 .

Comparaison entre Etats : Nous avons effectué une comparaison entre les taux bruts de scolarisation enregistrés en Algérie et ceux enregistrés dans certains pays, pour montrer les disparités qui existent entre eux, et ce en optant pour l'année scolaire 2009-2010, et nous avons fait ressortir ce qui suit :

Graph (6) : Comparaison des taux bruts de scolarisation au primaire et indice de parité en Algérie et certains pays.

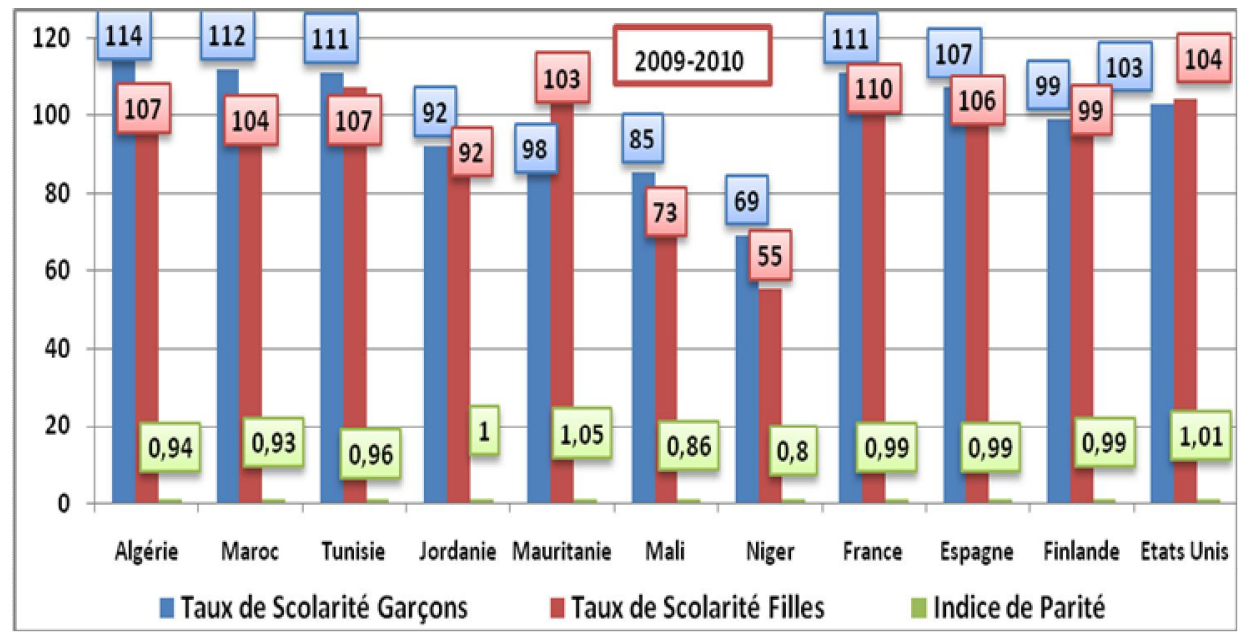

Source : Unesco (site UNESCO. org) (25) 
Il ressort de la comparaison que le taux brut de scolarisation des filles au primaire en Algérie est proche des taux enregistrés dans plusieurs pays arabes et européens et mieux que ceux enregistrés au Niger, Mali et en Jordanie. Quant à l'indice de parité entre sexes, il est moins que l'indice enregistré aux Etat Unis, en Finlande, en France, en Espagne, en Mauritanie, En Jordanie et en Tunisie.

\subsection{Taux brut de scolarisation en moyen}

Cet indicateur représente le nombre de la population scolarisée au primaire quel que soit leur âge (suivant les données fournies par les statistiques du ministère de l'éducation nationale, leur âge varient entre 10 et 19 ans) par rapport à la population algérienne de la catégorie d'âge de 12 à 15 ans représentant le nombre d'années passées par un élève au primaire (04 ans). Les résultats de cet indicateur sont les suivants :

Graph (7) : Evolution du taux brut de scolarisation en moyen et indice de parité entre sexes 
Mars. 2015. N 14

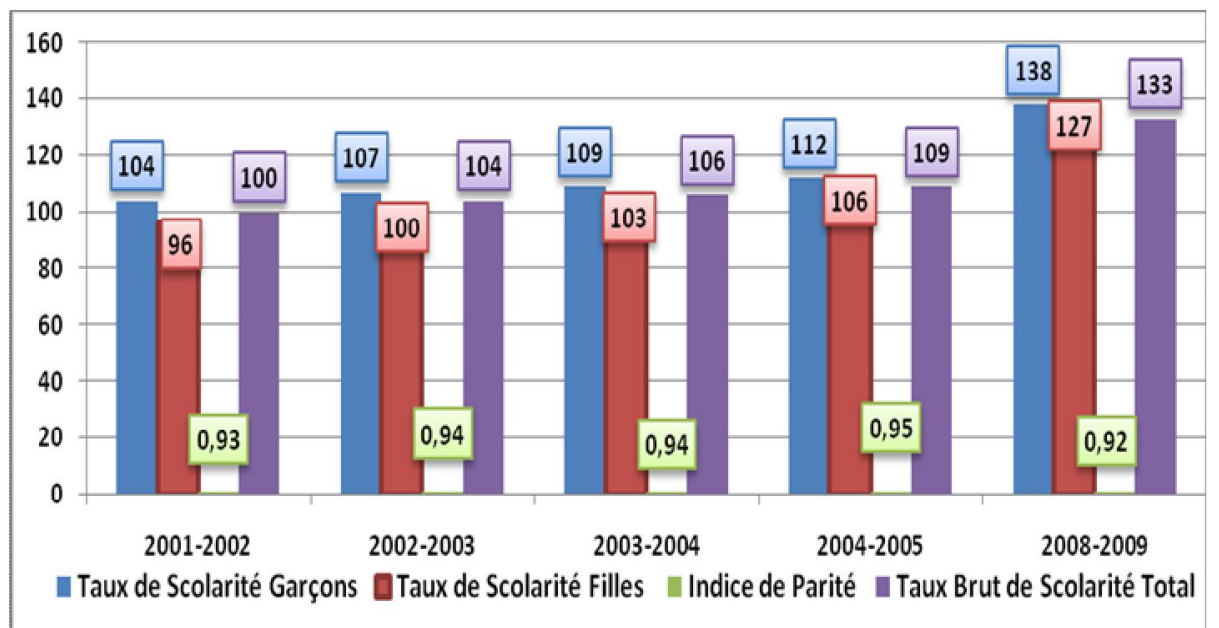

Source : Ministère de l'éducation nationale (26) (27) (28) (29)

Comparaison à travers le temps: Même observation pour le moyen, on constate que la scolarisation dans l'enseignement moyen en constante évolution durant ces dernières années; Le taux brut de scolarisation oscille entre $100 \%$ et $133 \%$ durant la période allant de 2001 à 2010 ; le taux de scolarisation des filles a augmenté de $96 \%$ à $127 \%$ et le taux de scolarisation des garçons de $104 \%$ à $138 \%$. Il y a lieu de signaler que le taux brut de scolarisation comprend les enfants dans les établissements d'enseignement moyen, dont l'âge varie, selon les statistiques, entre 12 et 19 ans; les élèves à l'âge avancé ne sont pas nombreux. L'indice de parité entre sexes a connu des hauts et des bas durant la même période.

Comparaison entre Etats : Nous avons effectué une comparaison entre les taux bruts de scolarisation enregistrés en Algérie et ceux enregistrés dans certains pays, pour montrer les disparités qui existent 
entre eux, et ce en optant pour l'année scolaire 2009-2010, et nous avons fait ressortir ce qui suit :

Graph (8) : Comparaison des taux bruts de scolarisation en moyen et indice de parité en Algérie et certains pays.

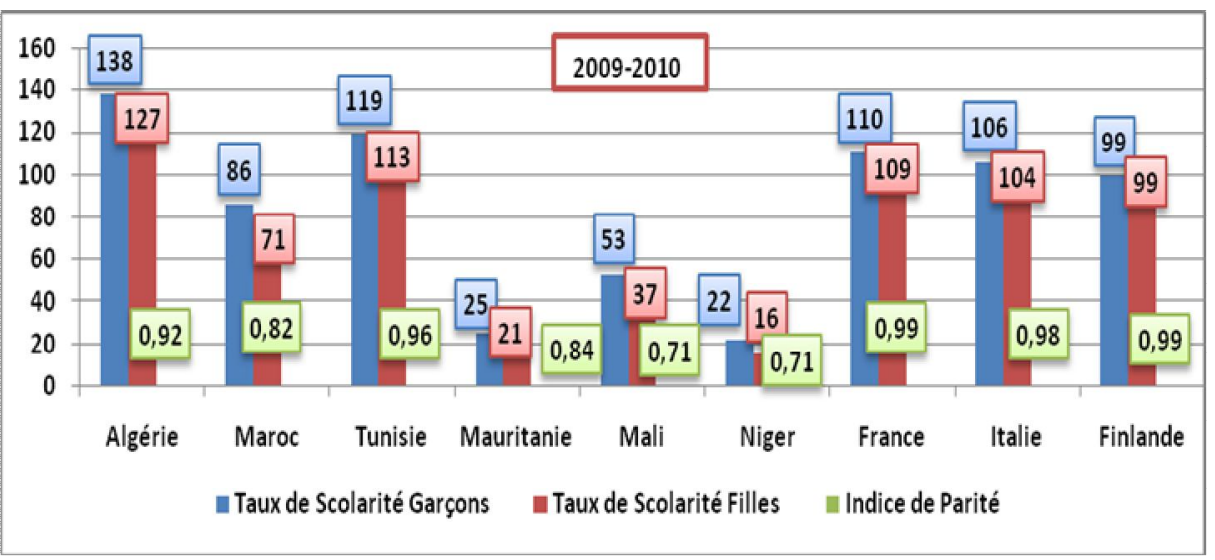

Source : Unesco (site UNESCO. org) (30)

Il ressort de la comparaison que le taux bruts de scolarisation des filles en moyen en Algérie est plus important par rapport à ceux enregistrés dans plusieurs pays. Quant à l'indice de parité entre sexes, il est moins que l'indice enregistré aux Etat Unis, en Finlande, en France, en Espagne et en Tunisie. 


\section{3 - Taux net de scolarisation :}

\subsection{Taux net de scolarisation au primaire :}

Cet indicateur représente le taux net de scolarisation selon l'âge de scolarisation au primaire (de 6 à 11 ans) par rapport à la population algérienne de la même catégorie d'âge (entre 06 et 11 ans). Les résultats de cet indicateur sont les suivants :

Graph (9): Evolution du taux net de scolarisation au primaire et indice de parité entre sexes

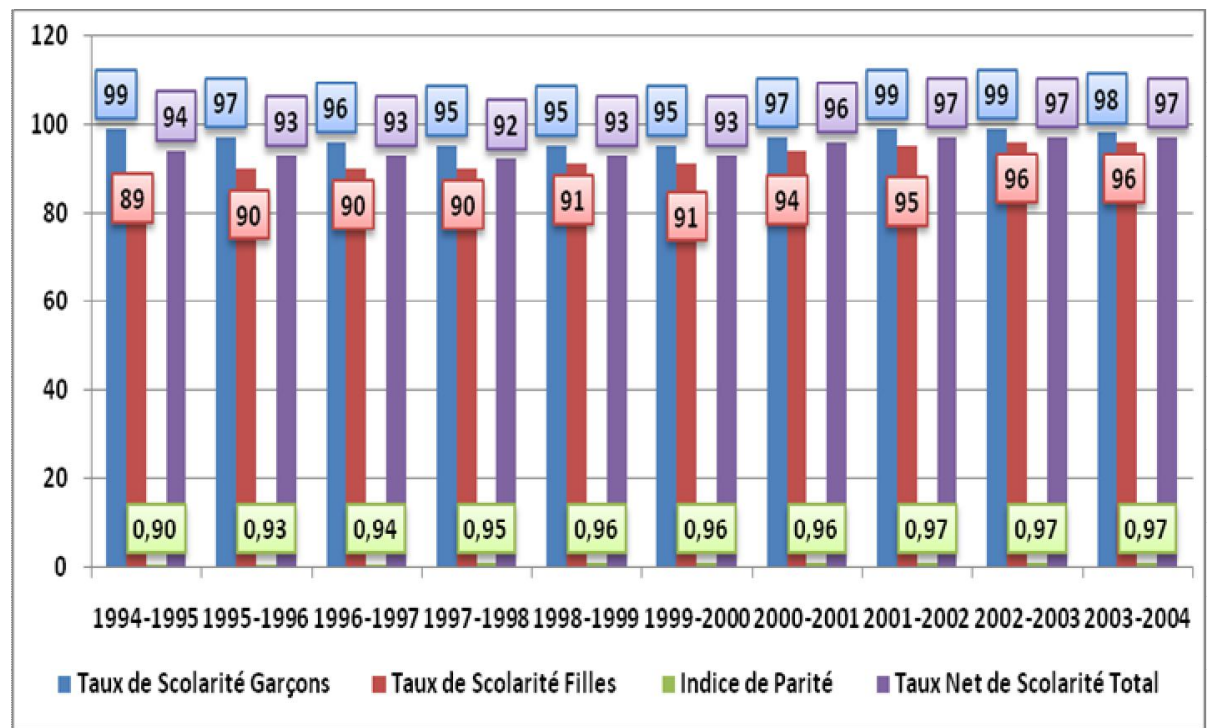

Source : Ministère de l'éducation nationale (31) (32) (33) (34)

Comparaison à travers le temps : La scolarisation des enfants au primaire était en constante augmentation durant la période allant de 1994 à 2004. Le taux de scolarisation des filles a augmenté de $89 \%$ à $96 \%$, par contre, le taux de scolarisation des garçons a oscillé entre 
augmentation et diminution de $95 \%$ et $99 \%$. L'indice de parité entre sexes a connu une augmentation de 0.90 à 0.97 .

Comparaison entre Etats : Nous avons effectué une comparaison entre les taux nets de scolarisation enregistrés en Algérie et ceux enregistrés dans certains pays, pour montrer les disparités qui existent entre eux, et ce en optant pour l'année scolaire 2009-2010, et nous avons fait ressortir ce qui suit :

Graph (10) : Comparaison des taux nets de scolarisation au primaire et indice de parité en Algérie et certains pays.

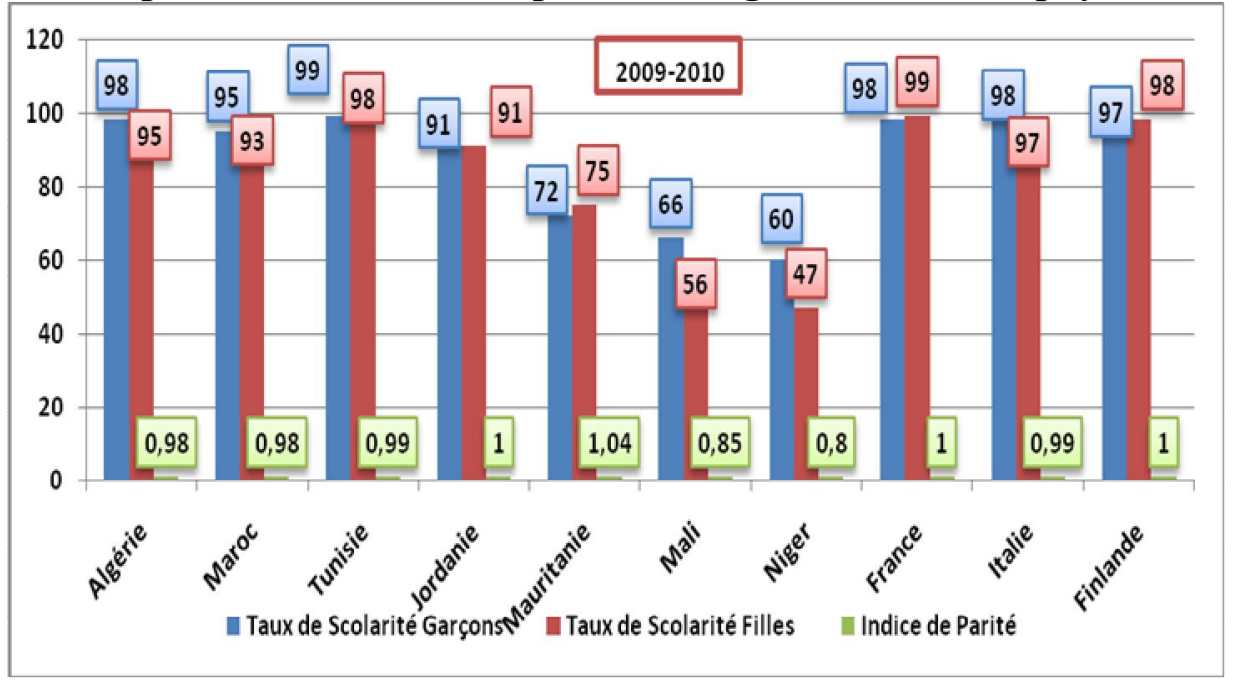

Source : Unesco (site UNESCO. org) (35)

Il ressort de la comparaison que le taux brut de scolarisation des filles de 06 à 11 ans au primaire en Algérie est moins que le taux enregistré en Finlande, en Italie, en France et en Tunisie et mieux que celui enregistré au Niger, au Mali et en Mauritanie. Quant à l'indice de 
parité entre sexes, il est moins que l'indice enregistré dans la majorité des pays européens et arabes.

\subsection{Taux net de scolarisation en moyen}

Cet indicateur représente le taux net de scolarisation en moyen (12 à 15 ans) par rapport à la population algérienne de la même catégorie d'âge (12 à 15). Les résultats de cet indicateur sont les suivants :

\section{Graph (11) : Evolution du taux net de scolarisation en moyen et indice de parité entre sexes}

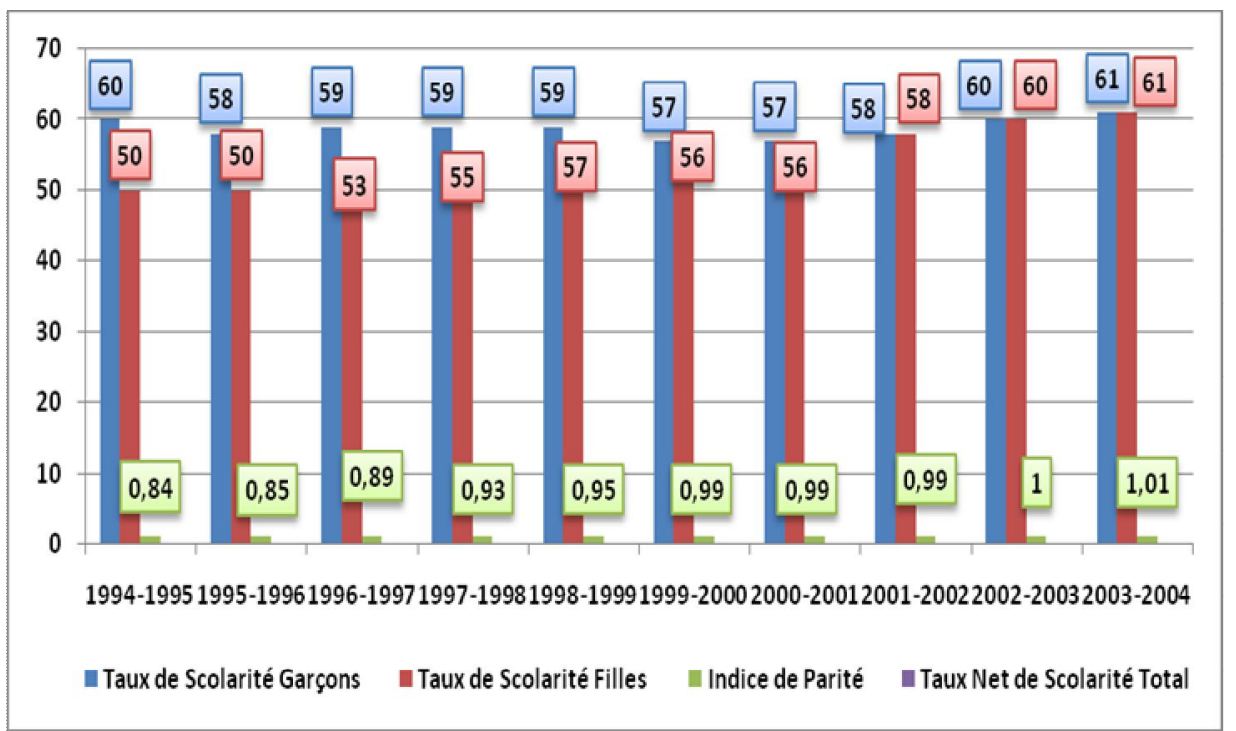

Source : Ministère de l'éducation nationale (36) (37) (38) (39)

Comparaison à travers le temps : On constate que les taux nets de scolarisation en moyen sont beaucoup moins élevés par rapport aux taux nets enregistrés au primaire ; le taux net de scolarisation oscille entre $56 \%$ et $61 \%$ durant la période allant de 1994 à 2004 ; le taux de 
scolarisation des filles a augmenté de $50 \%$ à $61 \%$; le taux de scolarisation des garçons a connu des hauts et des bas durant la même période. L'indice de parité entre sexes a augmenté de 0.84 à 1.01 .

\section{4- Taux de scolarisation selon les catégories d'âge :}

Nous avons opté pour l'année scolaire 2003-2004 pour calculer le taux de scolarisation en enseignement obligatoire selon les catégories d'âge(les données disponible), et nous avons abouti aux résultats suivants:

- Il y a un taux de $7 \%$ des enfants de 12 ans et un taux de $12 \%$ des enfants de 13 ans, qui ne sont pas scolarisés en enseignement obligatoire. il en va de même pour les enfants de 14 à 15 ans. En effet, un taux de $19 \%$ des enfants de 14 ans et un taux de $20 \%$ des enfants de 15 ans ne sont pas scolarisés et se trouvent en dehors de l'enseignement obligatoire et en dehors de l'enseignement secondaire.

- La question concernant ces enfants reste posée, sachant que le système offre à l'élève la possibilité de continué sa scolarité dans l'enseignement obligatoire, au motif, soit de scolarisation tardive ou redoublement de l'année ; il n'est exclu qu'après avoir atteint l'âge de 16 ans. Ceci témoigne de la déperdition importante de la catégorie d'âge de 12 à 15 ans, notamment en moyen.

\section{5- Le coefficient de scolarisation selon les wilayas, en enseignement obligatoire :}


A travers les indicateurs précédents nous avons pu connaitre les taux de scolarisation au niveau national, par rapport au nombre de la population algérienne dans les catégories d'âge scolarisables. Dans cette partie nous allons aborder le coefficient de scolarisation par régions ou par wilayas par rapport à l'ensemble de la population de chaque wilaya. Cet indicateur montre les disparités de chances à la scolarisation en enseignement obligatoire entre les wilayas. Pour cet indicateur le choix a été porté sur 03 années : 1990, 200 et 2003 (selon les données disponibles sur le nombre de la population de chaque wilaya). Les résultats sont récapitulés dans le tableau suivant :

Evolution du coefficient de scolarisation par wilaya dans le primaire et le moyen

\begin{tabular}{|c|c|c|c|c|c|c|}
\hline \multirow{2}{*}{$\begin{array}{c}\text { Wilay } \\
\text { as }\end{array}$} & \multicolumn{3}{|c|}{$\begin{array}{l}\text { Enseigneme } \\
\text { nt Primaire }\end{array}$} & \multicolumn{3}{|c|}{$\begin{array}{l}\text { Enseignem } \\
\text { ent Moyen }\end{array}$} \\
\hline & $\begin{array}{l}19 \\
90\end{array}$ & $\begin{array}{l}20 \\
00\end{array}$ & $\begin{array}{l}20 \\
03\end{array}$ & $\begin{array}{l}19 \\
90\end{array}$ & $\begin{array}{l}20 \\
00\end{array}$ & $\begin{array}{l}20 \\
03\end{array}$ \\
\hline Adrar & 15 & 17 & 15 & 3 & 6 & 7 \\
\hline Chelef & 17 & 17 & 16 & 5 & 6 & 7 \\
\hline laghou & 17 & 17 & 17 & 5 & 7 & 8 \\
\hline
\end{tabular}

\begin{tabular}{|c|c|c|c|c|c|c|}
\hline \multirow{2}{*}{ Wilayas } & \multicolumn{3}{|c|}{$\begin{array}{c}\text { Enseignem } \\
\text { ent } \\
\text { Primaire }\end{array}$} & \multicolumn{3}{|c|}{$\begin{array}{l}\text { Enseignem } \\
\text { ent Moyen }\end{array}$} \\
\hline & $\begin{array}{l}19 \\
90\end{array}$ & $\begin{array}{l}20 \\
00\end{array}$ & $\begin{array}{l}20 \\
03\end{array}$ & 19 & 20 & $\begin{array}{l}20 \\
03\end{array}$ \\
\hline $\begin{array}{c}\text { Constan } \\
\text { tine }\end{array}$ & 16 & 14 & 12 & 6 & 6 & 6 \\
\hline Médéa & 17 & 14 & 12 & 5 & 6 & 6 \\
\hline Mostaga & 15 & 14 & 13 & 5 & 6 & 6 \\
\hline
\end{tabular}


Revue des sciences de l'homme et de la société.......... Dr.Zoulikha Toutaoui

\begin{tabular}{|c|c|c|c|c|c|c|c|c|c|c|c|c|c|}
\hline at & & & & & & & nem & & & & & & \\
\hline \begin{tabular}{|c} 
Oum \\
EL \\
Bouag
\end{tabular} & 18 & 17 & 16 & 6 & 7 & 8 & M'sila & 18 & 17 & 16 & 5 & 7 & 7 \\
\hline Batna & 17 & 15 & 13 & 6 & 6 & 7 & Mascar & 16 & 12 & 12 & 5 & 5 & 5 \\
\hline Bejaia & 18 & 16 & 12 & 6 & 7 & 8 & Ourgla & 21 & 22 & 20 & 6 & 9 & 9 \\
\hline $\begin{array}{c}\text { Beskr } \\
\end{array}$ & 17 & 20 & 19 & 6 & 8 & 8 & Oran & 16 & 13 & 13 & 6 & 6 & 6 \\
\hline $\begin{array}{c}\text { Becha } \\
\text { r }\end{array}$ & 19 & 15 & 13 & 6 & 7 & 7 & $\begin{array}{c}\text { El } \\
\text { Bayadh }\end{array}$ & 14 & 14 & 15 & 5 & 6 & 6 \\
\hline Blida & 19 & 14 & 13 & 6 & 6 & 6 & Elizi & 18 & 22 & 24 & 4 & 7 & 8 \\
\hline Bouira & 16 & 14 & 12 & 5 & 7 & 7 & $\begin{array}{c}\text { Bordj } \\
\text { Bou } \\
\text { arreridj }\end{array}$ & 19 & 18 & 15 & 6 & 7 & 8 \\
\hline $\begin{array}{l}\text { Tama } \\
\text { nrasse }\end{array}$ & 13 & 17 & 16 & 3 & 6 & 7 & $\begin{array}{c}\text { Boumer } \\
\text { des }\end{array}$ & 18 & 11 & 9 & 6 & 5 & 5 \\
\hline Tébess & 17 & 18 & 17 & 6 & 7 & 8 & El-Taref & 18 & 13 & 12 & 7 & 6 & 6 \\
\hline
\end{tabular}


Mars. 2015. N 14

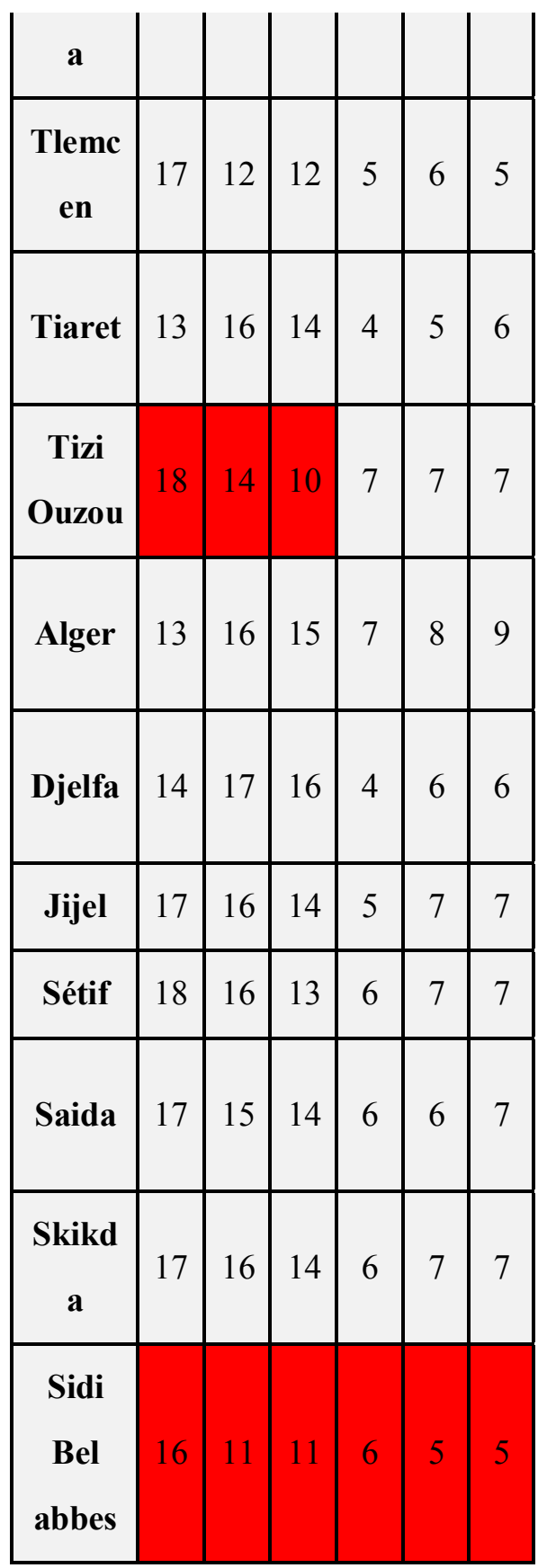

\begin{tabular}{|c|c|c|c|c|c|c|}
\hline Tindouf & 18 & 22 & 22 & 6 & 8 & 9 \\
\hline $\begin{array}{c}\text { Tissems } \\
\text { silt }\end{array}$ & 16 & 16 & 15 & 4 & 6 & 7 \\
\hline El-Oued & 18 & 19 & 18 & 5 & 8 & 8 \\
\hline $\begin{array}{c}\text { Khench } \\
\text { ela }\end{array}$ & 16 & 17 & 16 & 5 & 7 & 8 \\
\hline $\begin{array}{l}\text { Souk- } \\
\text { Ahras }\end{array}$ & 17 & 14 & 13 & 6 & 6 & 6 \\
\hline Tipaza & 18 & 10 & 9 & 6 & 4 & 4 \\
\hline Mila & 17 & 18 & 16 & 5 & 7 & 8 \\
\hline $\begin{array}{l}\text { Ain } \\
\text { Defla }\end{array}$ & 16 & 16 & 15 & 5 & 6 & 6 \\
\hline Naama & 16 & 14 & 14 & 6 & 7 & 7 \\
\hline $\begin{array}{c}\text { Ain } \\
\text { Témouc } \\
\text { hent }\end{array}$ & 16 & 12 & 12 & 6 & 5 & 6 \\
\hline
\end{tabular}



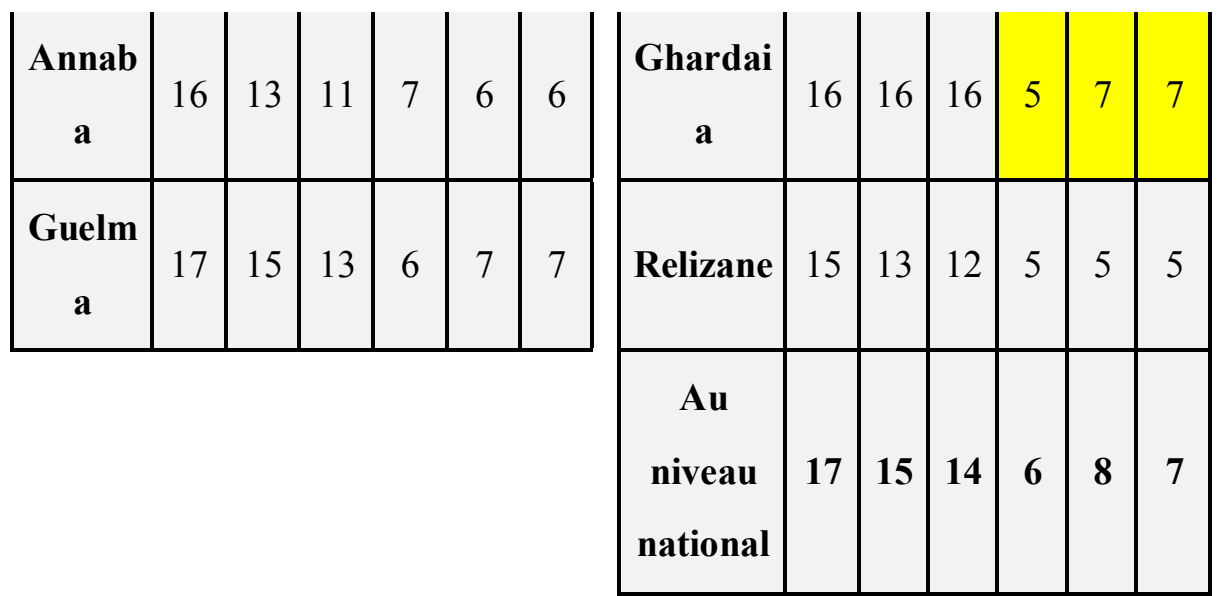

Source : Ministère de l'éducation nationale (40) (41) (42)

\section{Comparaison à travers le temps :}

- Enseignement primaire : La première observation à relever est la baisse du coefficient de scolarisation au primaire au niveau national depuis les années 90 (population scolarisée par rapport à l'ensemble de la population algérienne), cette diminution a été constatée dans 21 wilayas.

$>$ Le coefficient a baissé dans 06 wilayas depuis 1990, telle que :

Sid Belabes, Médéa, Ain Timouchent, baisse importante dans la wilaya de Tizi Ouzou (de $18 \%$ à $10 \%$ ), de Boumerdes (18 $\%$ à $9 \%$ ), de Tipaza ( $17 \%$ à $9 \%$ ), ces deux dernières wilayas ont enregistré le plus faible coefficient de scolarisation durant l'année 2003. 
$>$ Le coefficient le plus élevé a été enregistré dans les wilayas du sud, telle que : Illizi avec $24 \%$, Tindouf $22 \%$ ainsi que la wilaya de Ouargla.

- Enseignement moyen : il a connu une amélioration dans la majorité des wilayas; le coefficient de scolarisation a augmenté dans 32 wilayas. Le coefficient de scolarisation a baissé dans les wilayas de Sidi Bélabes, Oran, Boumerdes et Tipaza.

$>$ Une augmentation du coefficient de scolarisation a été relevée dans les wilayas du sud : Adrar (de $3 \%$ à $7 \%$ ), Illizi (de $4 \%$ à $8 \%$ ), Ouargla (de $6 \%$ à $9 \%$ ) Tindouf (de $6 \%$ à $9 \%$ ), El Oued (de $5 \%$ à $8 \%$ ) et Ghardaia (de $5 \%$ à $7 \%$ ).

$>$ Le plus faible coefficient de scolarisation en 2003 a été enregistré à Tipaza avec $4 \%$ et à Boumerdes avec $5 \%$, et le plus élevé a été enregistré à Ouargla avec $9 \%$, à Alger avec $9 \%$ et à Mila avec $8 \%$.

- Au niveau national: le coefficient de scolarisation en enseignement obligatoire au niveau national a été évalué à $22 \%$ en 1990, $21 \%$ en $2000,20 \%$ en 2003, une baisse contenue de la scolarisation dans ce palier est constatée. Cette baisse est peut être due à la baisse de la population scolarisable de 06 à 15 ans en général et de 6 à 11 ans en particulier (observation pour l'enseignement primaire). 


\section{Comparaison entre Etats :}

Nous procéderons à une comparaison avec certains pays pour déterminer le niveau du coefficient de scolarisation et les disparités qui y existent, dans l'enseignement obligatoire (primaire et moyen) par rapport à la catégorie d'âge (5 et 14 ans pour les états membres de l'OCDE et la catégorie d'âge de 6 à 15 ans pour l'Algérie).

Graph (12) : Comparaison des coefficients de scolarisation dans l'enseignement obligatoire en Algérie et d'autres pays membres de l'OCDE.

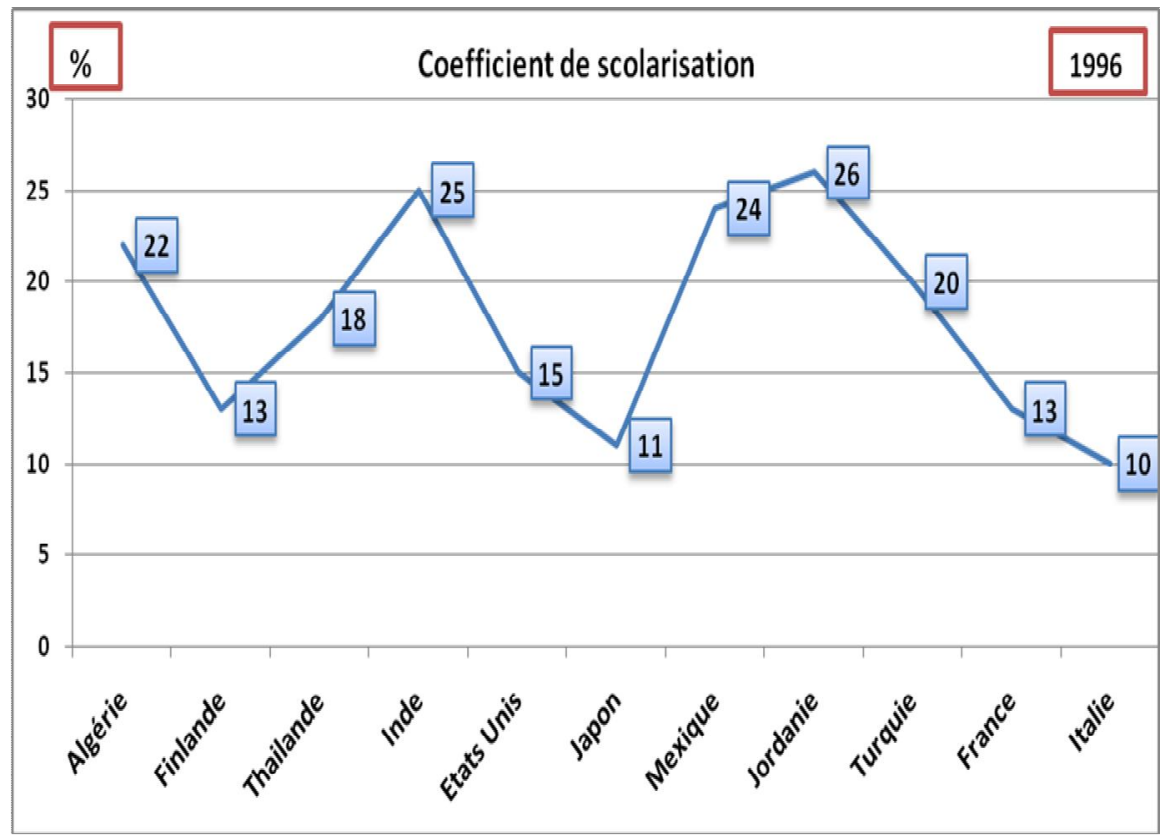

Source : OCDE (43) (44)

- En comparant avec certains pays membres de L'OCDE pour l'année 1996, on constate que le coefficient de scolarisation de la 
catégorie de 6 et 15 ans en Algérie, évalué à $22 \%$, n'est pas très loin des pays ayant une densité populaire. Il est élevé par rapport à tous les pays membres.

- Il y a lieu de signaler également que la baisse du coefficient de scolarisation constatée en Algérie pendant ces dernières années, est constatée aussi dans plusieurs pays. En effet, dans la majorité des pays membres de l'OCDE, une baisse de la population de 5 et 14 ans a été constatée entre 1990 et 1996, tel que : la Finlande et La France (-5\%), l'Italie, le Japon, l'Ireland et la Hongrie (entre $05 \%$ et $-10 \%$ ), l'Espagne (entre $-20 \%$ et $-25 \%$ ).

- Une augmentation de la population dans la même catégorie d'âge a été constaté dans d'autres pays, tel que: l'Autriche, L'Allemagne, la Suède, les Etats unis, (entre $+05 \%$ et $\quad+10 \%$ ), l'Australie, la Belgique, Le Danemark, le Mexique et la Norvège $(+5 \%)$.

- La catégorie d'âge de 6 et 14 ans a augmenté en Algérie de $+7 \%$ entre 1990 et 1996, et a augmenté de $+0.9 \%$ entre 1996 et 2000. Cependant cette catégorie d'âge a connu une baisse après l'an 2000 , et a baissé de (-5.5\%) entre 2000 et 2003.

\section{Conclusions générales :}

Au fil de temps, l'égalité entre les deux sexes au droit à l'enseignement obligatoire a été assez respectée. L'indice de parité entre sexes a été évalué à 0.98 pour les nouveaux admis au primaire ; le même taux a été enregistré pour les nouveaux admis 
à l'âge de six ans, qui est proche de l'indice enregistré dans certains pays.

Le taux de scolarisation des filles a dépassé 100\%; l'indice de parité entre sexes a augmenté de $0.94 \%$ pour la population scolarisée au primaire, mais moins que le taux enregistré dans certains pays. Quant à l'enseignement en moyen, l'indice de parité a atteint 1.01. Une baisse de scolarisation des garçons continue a été enregistrée depuis les années 90 dans ce palier.

Les taux de scolarisation ont montré que $7 \%$ des enfants de 12 ans et $12 \%$ des enfants de 13 ans ne sont pas scolarisés en enseignement obligatoire. Il en va de même pour les enfants de 14 à 15 ans. En effet, un taux de $19 \%$ des enfants de 14 ans et un taux de $20 \%$ des enfants de 15 ans ne sont pas scolarisés et se trouvent en dehors de l'enseignement obligatoire et en dehors de l'enseignement secondaire.

La question concernant ces enfants reste posée, sachant que le système offre à l'élève la possibilité de continué sa scolarité dans l'enseignement obligatoire, au motif, soit de scolarisation tardive ou redoublement de l'année; il n'est exclu qu'après avoir atteint l'âge de 16 ans. Ceci témoigne de la déperdition importante de la catégorie d'âge de 12 à 15 ans, notamment en moyen. 
Le coefficient de scolarisation par wilaya, concernant les chances des enfants à l'enseignement obligatoire, a montré une baisse dans le coefficient de scolarisation au primaire depuis les années 90 et ce dans 21 wilayas, une amélioration en moyen dans la plupart des wilayas du pays, et une augmentation de ce coefficient dans certaine wilayas du sud. Les Wilaya de Tizi Ouzou, Tipaza et Boumerdes ont connu une baisse en matière de scolarisation des enfants au primaire te en moyen.

Alors, quels sont les raisons de la baisse de scolarisation dans ces régions? Cela est-il dû à la baisse du nombre d'enfant scolarisable en raison des décès ou à la baisse des naissances? Ou serait-il dî̀ à l'abandon de l'école par les enfants pour aider leurs familles ou à la situation sécuritaire dans ces régions? 


\section{Références}

(1) Ministère de l'éducation et de la formation. évaluation du système éducatif (1962-1998) : L'enseignement fondamental,_Algérie, (mars 1998) .

(2) Ministère de l'éducation nationale, bulletin officiel.. L'organisation de l'éducation et la formation en Algérie : ordonnance n³5-76 du 16 Avril. (1995) ,P $18-21$.

(3) Présidence de la République Algérienne. le Système Educatif: Bilan et Perspectives. Alger. .(Juin 1993), PP 11- 13.

.(4) Ministère de l'éducation nationale, bulletin officiel. (1995). L'organisation de $\underline{\text { l'éducation et la formation en Algérie : ordonnance n³5-76 du } 16 \text { Avril. }}$

(5) Présidence de la République Algérienne, Idem , P 54.

(6) Ministère de l'éducation nationale, bulletin officiel.. Textes sur la réforme du système éducatif. $\mathrm{N}^{\circ}$ spécial. O.N.P.S Algérie_(janvier 2005)

(7) Idem, PP 3-14.

(8) Sauvageot, C. Les indicateurs pour la planification de l'éducation : un guide pratique. IIPE ,Paris , (1996).

.(9) UNESCO, Développement d'indicateurs pour la planification de l'éducation en Afrique francophone de l'Ouest, IIPE, Paris , (1998).

(10) Jarousse, J, P. et Mingat,A , Evaluation globale de la politique éducative marocaine. IREDU-CNRS, Université de Bourgogne, France, (juin 1992).

(11) Ministère de l'éducation nationale, direction de planification, sous-direction des statistiques Série statistique de 1962 à 1998. Idem. 
(12) Ministère de l'éducation nationale, direction de planification, sous-direction des statistiques, bulletin des statistiques. O.N.P.S, Algérie

(13) Office National des Statistiques..Population Algérienne résidente, Prévision 1977/2008. Collections statistiques, ONS, Alger. (1980)

(14)Office National des Statistiques. Démographie: Projections de population 1990/2020. Collections statistiques N66, ONS ,Alger . (1994).

(15) Unesco. Tableaux statistiques. www.UNESCO.Org/Education/

(16) Ministère de l'éducation nationale, direction de planification, sous-direction des statistiques Série statistique de 1962 à 1998, Idem.

(17) Ministère de l'éducation nationale, direction de planification, sous-direction des statistiques, bulletin des statistiques. Idem.

(18) Office National des Statistiques. Population Algérienne résidente, Prévision 1977/2008.Idem.

(19)Office National des Statistiques. Démographie: Projections de population 1990/2020.Idem.

(20)Unesco. Tableaux statistiques.Idem .

(21) Ministère de l'éducation nationale, direction de planification, sous-direction des statistiques Série statistique de 1962 à 1998 . Idem.

(22) Ministère de l'éducation nationale, direction de planification, sous-direction des statistiques, bulletin des statistiques. Idem.

(23) Office National des Statistiques. Population Algérienne résidente, Prévision 1977/2008.Idem.

(24)Office National des Statistiques. Démographie: Projections de population 1990/2020. Idem. 
(25)Unesco. Tableaux statistiques. Idem.

(26) Ministère de l'éducation nationale, direction de planification, sous-direction des statistiques Série statistique de 1962 à 1998. Idem.

(27) Ministère de l'éducation nationale, direction de planification, sous-direction des statistiques, bulletin des statistiques. Idem.

(28) Office National des Statistiques. Population Algérienne résidente, Prévision 1977/2008.Idem.

(29) Office National des Statistiques. Démographie: Projections de population 1990/2020. Idem.

(30)Unesco. Tableaux statistiques .Idem.

(31) Ministère de l'éducation nationale, direction de planification, sous-direction des statistiques Série statistique de 1962 à 1998. Idem.

(32) Ministère de l'éducation nationale, direction de planification, sous-direction des statistiques, bulletin des statistiques Idem.

(33) Office National des Statistiques. Population Algérienne résidente, Prévision 1977/2008.Idem

(34) Office National des Statistiques. Démographie: Projections de population 1990/2020. Idem.

(35)Unesco. Tableaux statistiques. Idem.

(36) Ministère de l'éducation nationale, direction de planification, sous-direction des statistiques Série statistique de 1962 à 1998 . Idem.

(37) Ministère de l'éducation nationale, direction de planification, sous-direction des statistiques, bulletin des statistiques Idem. 
(38) Office National des Statistiques. Population Algérienne résidente, Prévision 1977/2008.Idem.

(39) Office National des Statistiques. Démographie: Projections de population 1990/2020. Idem.

(40) Ministère de l'éducation nationale, direction de planification, sous-direction des statistiques Série statistique de 1962 à 1998. Idem.

(41) Ministère de l'éducation nationale, direction de planification, sous-direction des statistiques, bulletin des statistiques Idem.

(42) Office National des Statistiques. Démographie: Projections de population à l’horizon 2030. Collections statistiques $\mathrm{N}^{\circ} 106$, ONS, Alger . (2004).

(43) OCDE-CRIE. (1995). Regard sur l'éducation: Les indicateurs de l'OCDE. Centre de recherche et l'innovation dans l'enseignement. Paris : OCDE-CRIE. PP 57-64.

(44) OCDE-CRIE. (1998). Regards sur l'éducation : les indicateurs de l'OCDE. Centre de recherche et l'innovation dans l'enseignement Paris : OCDE-CERI. P 13. 